\title{
Qualitative and quantitative analyses of aconite alkaloids in Aconiti kusnezoffii Radix, and NO inhibitory activity evaluation of the alkaloid extracts
}

\author{
Qin-Yu LI' ${ }^{1}$, Bo WU ${ }^{2}$, Xue GONG ${ }^{1}$, Wen-Shuo WANG ${ }^{1}$, Jia-Xin ZHU ${ }^{1}$, Chun-Hong ZHANG ${ }^{1,3,4}$ and \\ Na ZHANG ${ }^{1,3,4 *}$ (D)
}

\begin{abstract}
Aconiti kusnezoffii Radix (Caowu), a traditional Chinese medicine and Mongolian medicine, has been commonly used owing to its analgesic and anti-inflammatory effects. Here, we performed a qualitative analysis of aconite alkaloids (AAs) in raw and processed Aconiti kusnezoffii Radix by ultra-high-performance liquid chromatography (HPLC) electrospray ionizationtandem mass spectrometry. Furthermore, we quantified benzoylaconine, benzoylmesaconine, benzoylhypaconine, aconitine, mesaconitine, and hypaconitine in raw and processed Aconiti kusnezoffii Radix using a HPLC system coupled with a diode array detector. The analysis of AA fragmentation patterns in positive electrospray ionization mode revealed $155 \mathrm{AAs}$, including 6 with unambiguous identities, 109 with putative assignments, and 40 unknown compounds. Additionally, lipopolysaccharide-treated RAW264.7 macrophages were used to evaluate the anti-inflammatory properties of the alkaloid extracts of Aconiti kusnezoffii Radix. Importantly, alkaloid extracts of Aconiti kusnezoffii Radix at three different concentrations exhibited protective effect against lipopolysaccharide-induced inflammation. Thus, the proposed method can be used not only for the qualitative and quantitative analyses of Aconiti kusnezoffii Radix, but also for evaluating other Aconitum spp. Our study lays a foundation for the clinical application of poisonous Aconitum.
\end{abstract}

Keywords: aconite alkaloid; Aconiti kusnezoffii Radix; anti-inflammatory effect; qualitative and quantitative analyses; ultrahigh performance liquid chromatography-electrospray ionization tandem mass spectrometry.

Practical Application: Qualitative and quantitative analysis and activity evaluation of aconitum alkaloids.

\section{Introduction}

Aconiti kusnezoffii Radix is a traditional Chinese medicine (TCM) (commonly called "Caowu") (National Pharmacopoeia Committee, 2015) and Mongolian medicine (commonly called "Benga") (Ao \& Buhe, 2013). In both traditional medicinal systems, Aconiti kusnezoffii Radix is commonly used owing to its analgesic (Liu et al., 2013) and anti-inflammatory effects(Li et al., 2019). The main active constituents of Aconitum spp. are aconite alkaloids (AAs), which have a C18-, C19-, or C20-diterpenoid skeleton (Wang et al., 2009; Wang et al., 2010; Wang \& Liang, 2002), and lipo-alkaloids (Borcsa et al., 2011), which are a type of C19-norditerpenoid alkaloids; these AAs are both active and toxic. The effective therapeutic doses of AAs are close to their toxic doses (Wang et al., 2018b). During processing, these alkaloids retain their analgesic properties, although their toxicity is reduced by approximately 100-fold (Zhang et al., 2015; Zhi et al., 2020).

To ensure the safety and effectiveness of AAs in clinical application, it is imperative to develop convenient, quick, and effective methods to identify and characterize AAs in raw and processed Aconiti kusnezoffii Radix. Liquid chromatography
(LC) has a better separation ability and high-resolution mass spectrometry (MS) has stronger analysis and identification abilities. MS/MS can provide extensive information of fragment ions, which is of significance for the identification of complex and same-type compounds. LC-MS/MS can effectively separate and help identify the chemical components of TCM (Pang et al., 2016; Song et al., 2015).

Currently, there is a lack of comprehensive qualitative and quantitative analyses of the chemical constituents of Aconiti kusnezoffii Radix. Therefore, we used an HPLC-ESI MS/MS and HPLC-DAD approach to investigate the types and content of components in raw and processed Aconiti kusnezoffii Radix. We then treated Raw264.7 cells with lipopolysaccharides (LPS) to establish a cell inflammatory reaction model (Wang et al., 2018a; Wang et al., 2016), and the release of nitric oxide (NO) was measured by adding alkaloid extracts of Aconiti kusnezoffii Radix (AECs) at different concentrations. This model can be tentatively used to evaluate the anti-inflammatory mechanism of AECs. 


\section{Materials and methods}

\subsection{Chemicals and reagents}

Raw and processed Aconiti kusnezoffii Radix samples were collected from different provinces in China and were authenticated by Prof. Chunhong Zhang, Baotou Medical College (Baotou, China). Six alkaloid reference standards, namely, BAC, BMA, BHA, AC, MA, and HA, were purchased from Shanghai Standard Biotech Co., Ltd. (Shanghai, China). The chemical structure of the six alkaloids is shown in Figure 1.

LC/MS-grade acetonitrile, methanol, and formic acid were purchased from Fisher Scientific (Shanghai, China). Other reagents used in this study were dimethyl sulfoxide (DMSO), penicillin, streptomycin (Haikelong Biochemical Products Co., Ltd., Beijing, China), Dulbecco's modified Eagle's medium (DMEM) high glucose (Gibco Life Technologies, CA, Carlsbad, USA), Sijiqing fetal bovine serum (FBS; Tianhang Biotechnology Co., Ltd., Zhejiang, China), MTT, and LPS (Sigma, St. Louis, MO, USA). All other reagents were of analytical grade and were provided by Beijing Chemical Works (Beijing, China).

\subsection{Sample preparation and extraction}

Stock solutions of the six alkaloid reference standards were prepared with DMSO, and then diluted with $70 \%$ methanol to obtain the following concentrations: $5.85 \mathrm{mg} / \mathrm{mL}$ BAC, $2.18 \mathrm{mg} / \mathrm{mL}$ BMA, $2.24 \mathrm{mg} / \mathrm{mL}$ BHA, $2.79 \mathrm{mg} / \mathrm{mL} \mathrm{AC}$, $1.56 \mathrm{mg} / \mathrm{mL} \mathrm{MA}$, and $3.93 \mathrm{mg} / \mathrm{mL}$ HA. The stock solution was stored at $4{ }^{\circ} \mathrm{C}$ until use.

All Aconiti kusnezoffii Radix samples were air-dried, ground, and sieved (60 mesh) to obtain a homogeneous powder. Five volumes of $70 \%$ methanol $(\mathrm{g} / \mathrm{mL})$ was added to $10 \mathrm{~g}$ of the powder, and the samples were soaked for $1 \mathrm{~h}$ and extracted for $30 \mathrm{~min}$ using an ultrasonicator $(250 \mathrm{~W}, 40 \mathrm{kHz})$. After cooling the sample to $25^{\circ} \mathrm{C}$, the lost weight was replenished with $70 \%$ methanol. Finally, each extract was filtered through a $0.22-\mu \mathrm{m}$ nylon membrane before HPLC-DAD and UHPLC Q-Exactive Orbitrap MS/MS analyses.

The five raw Aconiti kusnezoffii Radix samples were powdered to a homogeneous size using a mill, sieved (60 mesh), and further dried at $60{ }^{\circ} \mathrm{C}$ in an oven for $6 \mathrm{~h}$ to a constant weight. Each powdered sample ( $40 \mathrm{~g}$ ) was mixed and macerated with $1000 \mathrm{~mL}$ of $70 \%$ methanol for $24 \mathrm{~h}$. The extracted solution was filtered. The first filtered residue was extracted with $400 \mathrm{~mL}$ of $70 \%$ methanol for $30 \mathrm{~min}$ by ultrasonic treatment. The extracted solution was filtered, and the residue was extracted with $300 \mathrm{~mL}$ of $70 \%$ methanol by Soxhlet extraction for $2 \mathrm{~h}$. The three filtrates were combined, and the methanol was recovered by rotary evaporation and cooled at $4{ }^{\circ} \mathrm{C}$ for $24 \mathrm{~h}$. Thereafter, $250 \mathrm{~mL}$ of water was added to the extract at $25^{\circ} \mathrm{C}$, and the $\mathrm{pH}$ of the solution was adjusted to 1-2 with dilute hydrochloric acid and then to 9-10 with ammonia water. This $\mathrm{pH}$-adjusted solution was extracted three times with an equal volume of dichloromethane, yielding total alkaloids (AEC, $3.8 \mathrm{~g}$ ) and water (non-AEC, $32.8 \mathrm{~g}$ ).

\subsection{Instrumentation and operation conditions}

A UHPLC system, including an on-line degasser, a column oven, an autosampler, and a diode array detector (Thermo Fisher Scientific, Bremen, Germany), was used for quantitative data acquisition. A UHPLC system (Ultimate 3000) coupled with Q-Exactive Orbitrap MS (Thermo Fisher Scientific) was used for qualitative data acquisition.

The qualitative and quantitative methods are shown in Table 1. First, the qualitative analysis was performed to identify alkaloids in raw and processed Aconiti kusnezoffii Radix. A linear gradient elution program was developed as follows: 0-8 min, $5 \%$ B; 8-38 min, 5\%-95\% B; 38-45 min, 95\% B. Second, the quantitative analysis was simultaneously performed to assess the six alkaloids in raw and processed Aconiti kusnezoffii Radix. The eluting conditions were optimized as follows: $0-13 \mathrm{~min}$ : 5\%-25\% B, 13-30 min: 25\%-40\% B. An ultraviolet absorption wavelength of $230 \mathrm{~nm}$ was used to determine these six alkaloids.
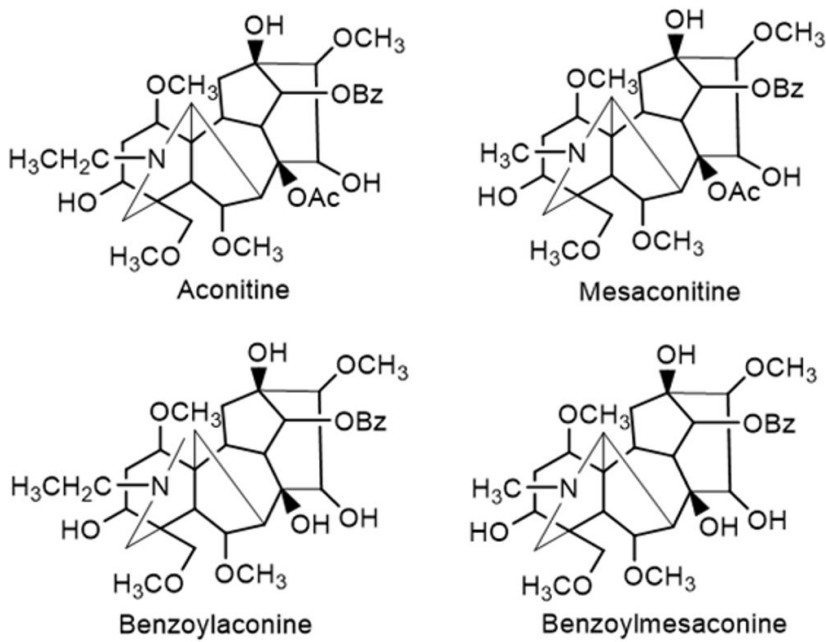

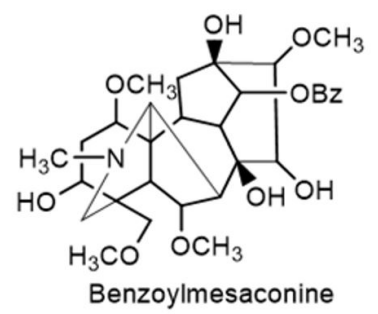

Benzoylmesaconine
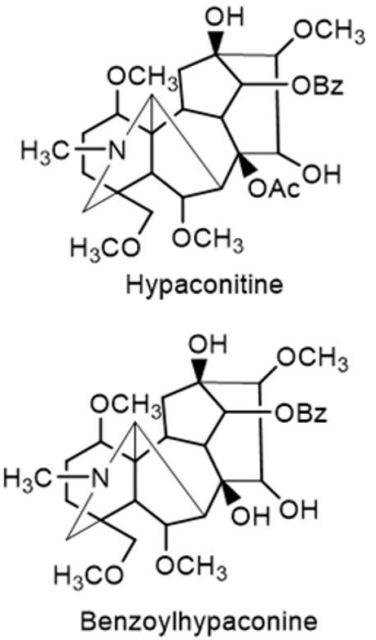

Figure 1. Chemical structure of the six alkaloid reference standards. 
Table 1. Qualitative and quantitative conditions (column, column temperature, flow rate, injection volume, and mobile phases A and B).

\begin{tabular}{|c|c|c|}
\hline \multirow{3}{*}{ Column } & Qualitative & Quantitative \\
\hline & $\begin{array}{c}\text { Thermo Hypersil } \\
\text { GOLD C18 }\end{array}$ & $\begin{array}{l}\text { ACE UltraCore } \\
\text { Super C18 }\end{array}$ \\
\hline & $\begin{array}{c}(1.9 \mu \mathrm{m}, 2.1 \mathrm{~mm} \times \\
100 \mathrm{~mm})\end{array}$ & $\begin{array}{c}(2.5 \mu \mathrm{m}, 4.6 \mathrm{~mm} \times \\
50 \mathrm{~mm})\end{array}$ \\
\hline Column Temp & $30^{\circ} \mathrm{C}$ & $30^{\circ} \mathrm{C}$ \\
\hline Flow rate $(\mathrm{mL} / \mathrm{min})$ & 0.3 & 1.5 \\
\hline Injection Volume & $2 \mu \mathrm{L}$ & $10 \mu \mathrm{L}$ \\
\hline Mobile Phase A & $\begin{array}{l}0.05 \% \text { formic acid in } \\
\text { water }\end{array}$ & $\begin{array}{l}5 \mathrm{mM} \text { ammonium } \\
\text { acetate in water } \\
\text { (pH 9.5, ammonia) }\end{array}$ \\
\hline Mobile Phase B & $\begin{array}{l}0.05 \% \text { formic acid in } \\
\text { acetonitrile }\end{array}$ & Acetonitrile \\
\hline
\end{tabular}

At the end of each run, the initial composition of the mobile phase $(5 \% \mathrm{~B})$ was allowed to run for $10 \mathrm{~min}$ to re-equilibrate the entire system.

\subsection{Optimization of the MS system}

A Q-Exactive Orbitrap MS/MS system equipped with heated ESI was used. As alkaloids were determined in the positive ion mode, the mass axis of the instrument was calibrated with positive ion mass calibration solution before each experiment. The optimum operating conditions are shown in Table 2. All parameters of the HPLC-MS system were controlled using Thermo Scientific TraceFinder software version 3.2 (Thermo Scientific).

\subsection{Anti-inflammatory assay}

Thermo Form 311 CO2 incubator (Thermo Fisher Scientific), SW-CJ ultra-clean table (Shanghai Xinmiao Medical Equipment Manufacturing Co., Ltd.), XDS-1B inverted microscope (Shanghai Beimu Instrument Factory), Epoch Enzyme Label Instrument (BioTek Instruments Co., Ltd., USA), and desktop centrifuge (Thermo Fisher Scientific) were used in the NO inhibition assay, as described below.

\section{Cell culture and viability}

RAW264.7 cells (Shanghai Institute of Cell Biology, Chinese Academy of Sciences) were cultured in DMEM containing 10\% heat-inactivated FBS, $100 \mathrm{U} / \mathrm{mL}$ penicillin, and $100 \mu \mathrm{g} / \mathrm{mL}$ streptomycin at $37^{\circ} \mathrm{C}$ with $5 \% \mathrm{CO} 2$. Logarithmic phase cells were used in the follow-up experiments.

Step 1: Cell viability was evaluated using RAW264.7 cells plated at a concentration of 105 cells $/ \mathrm{mL}$ in a 96 -well plate. Step 2: After the cells attached to the wall, they were treated with 0,25 , $50,100,200$, or $400 \mu \mathrm{g} / \mathrm{mL}$ AECs and non-AECs. Simultaneously, the normal control group (without AECs and non-AECs) was established. Step 3: The experiment was repeated in triplicate. After $24 \mathrm{~h}$ of culture, freshly prepared $5 \mathrm{mg} / \mathrm{mL}$ MTT solution $(10 \mu \mathrm{L})$ was added to each well, and the plate was incubated at $37^{\circ} \mathrm{C}$ for $4 \mathrm{~h}$. Step 4: At the end of cell culture, the supernatant from each well was removed and $150 \mu \mathrm{L}$ of DMSO was added to each well. Step 5: For cell viability analysis, the absorbance
Table 2. Ion source, full MS, and dd-MS2 parameter of the Q-Exactive Orbitrap MS/MS.

\begin{tabular}{lc}
\hline & Ion source \\
\hline Sheath gas pressure & $35 \mathrm{psi}$ \\
Auxiliary gas flow rate & $10 \mathrm{~L} / \mathrm{min}$ \\
Spare gas flow rate & $1 \mathrm{~L} / \mathrm{min}$ \\
Spray voltage & $+3500 \mathrm{~V}$ \\
Capillary temperature & $320^{\circ} \mathrm{C}$ \\
Probe heater temperature & $350^{\circ} \mathrm{C}$ \\
S-Lens RF level & $50 \mathrm{~V}$ \\
Full MS & \\
Resolution & 70000 \\
Mass range & $150-1500$ \\
AGC target & $3 \mathrm{e}^{6}$ \\
Maximum IT & $100 \mathrm{~ms}$ \\
dd-MS2 & \\
Resolution & 17500 \\
AGC target & $1 \mathrm{e}^{5}$ \\
Maximum IT & $50 \mathrm{~ms}$ \\
Loop count & 5 \\
Mass isolation window & $4.0 \mathrm{~m} / \mathrm{z}$ \\
Normalized collision energy & $30 \%$ \\
Minimum AGC & $8 \mathrm{e}^{3}$ \\
Intensity thresh & $1.5 \mathrm{e}^{5}$ \\
Exclude isotope & on \\
Dynamic exclusion & $10 \mathrm{~s}$ \\
\hline
\end{tabular}

(OD $570 \mathrm{~nm}$ ) of sample in each well was measured using an enzyme-labeled instrument after 10 min of shock treatment.

Cell survival rate $(\%)=\left(\begin{array}{l}O D \text { treatment well }- \\ \text { OD background control well }\end{array}\right) \times$

$100 /($ OD control well - OD background control well)

\section{NO assay}

The Griess reaction was used to assess NO production in cells. RAW264.7 cells were seeded in 96-well plates and allowed to adhere to the wall for $24 \mathrm{~h}$. The experimental and treatment groups were as follows: (1) the cells in the blank control group were cultured for $24 \mathrm{~h}$ without any treatment; (2) the cells in the model control group were treated with $1 \mu \mathrm{g} / \mathrm{mL}$ LPS for $24 \mathrm{~h}$; and (3) the cells in the experimental group were treated with $1 \mu \mathrm{g} / \mathrm{mL}$ LPS and AEC and non-AEC at different concentrations $(6.25,12.5,25$, and $50 \mu \mathrm{g} / \mathrm{mL})$ for $4 \mathrm{~h}$. Subsequently, cell culture and viability steps 3,4 , and 5 in section 3.5.1. were used.

$$
\text { NO inhibition rate }(\%)=\left(\begin{array}{l}
\text { OD model } \\
\text { control group }- \\
\text { OD experimental group }
\end{array}\right) \times 100 /\left(\begin{array}{l}
\text { OD model } \\
\text { control group }- \\
\text { OD blank } \\
\text { control group }
\end{array}\right)
$$

\section{Results}

\subsection{Qualitative analysis}

Through previous and preliminary study findings (Yue et al., 2009; Liang et al., 2016; Zhang et al., 2019; Zhi et al., 2020; Zhang et al., 2016; Xu et al., 2014), the positive ion mode was 
found to be more suitable for the detection of alkaloids and the stable detection of fragment ions with sufficient abundance. The normalized collision energy (NCE) mode was chosen as there is no need to optimize the collision energy. Approximately 155 peaks were detected within $40 \mathrm{~min}$ in the mass spectrum of the $70 \%$ methanol extract of raw and processed Aconiti kusnezoffii Radix (Figure 2A and 2B) in the positive ion mode. The compounds were tentatively identified based on the HPLC retention time ( $t_{R}$ in Supplementary Materials Table S2). The 155 compounds were identified by elemental composition of MS, and the MS/MS fragment ion data were compared with data available in the literature, using mzCloud and chemSpider.

The data of the identified AAs, including the $t_{R}$, elemental composition, calculated molecular mass, measured molecular mass, error (ppm), MS/MS information, and identification results are presented in Table S2. These data were used for predicting the AAs in raw and processed Aconiti kusnezoffii Radix. The mass error of $\mathrm{M}+\mathrm{H}]^{+}$was less than $5 \mathrm{ppm}$, which indicated that the measured molecular formula matched the calculated molecular mass. The reliability of elemental composition was relatively higher, which is a prerequisite for identification.

\subsection{Quantitative analysis}

\section{Validation of the quantitative analytical method}

Stock solutions of the six reference standards (benzoylaconine $\mathrm{BAC}]$, benzoylmesaconine BMA], benzoylhypaconine BHA], aconitine $\mathrm{AC}$ ], mesaconitine $\mathrm{MA}$ ], and hypaconitine $\mathrm{HA}$ ]), were prepared and diluted 2-8-fold for constructing calibration curves.

The linear range, linearity equation, correlation coefficient, limit of detection, and limit of quantification for the quantitative analysis of the six reference alkaloids are shown in Table 3.
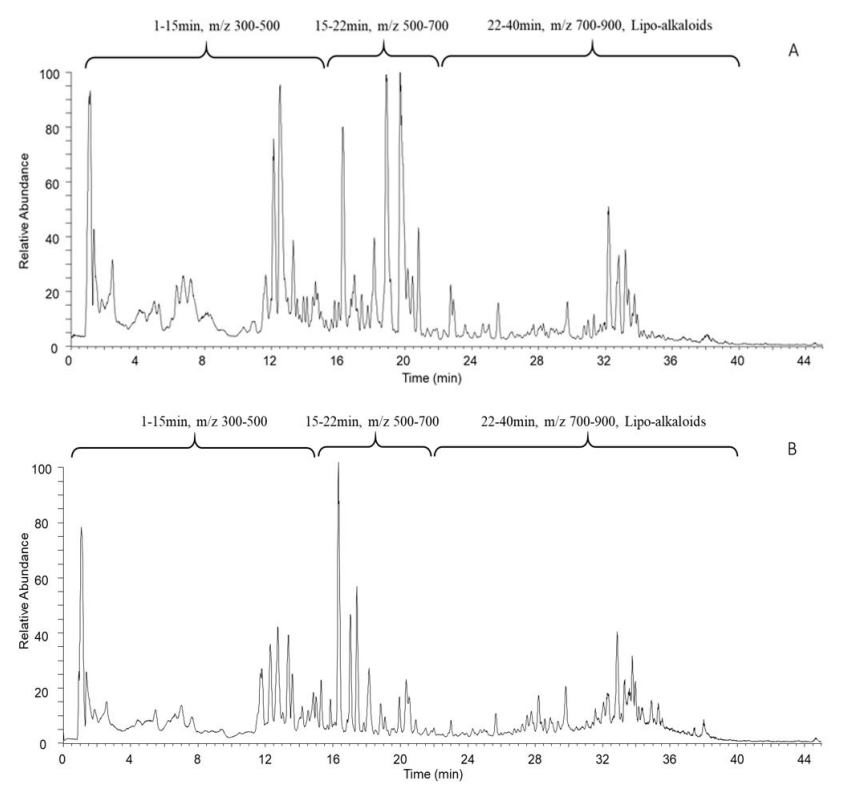

Figure 2. Total ion chromatograms (TIC) of the raw (A) and processed (B) Aconiti kusnezoffii Radix in the positive ionization mode.
The precision, repeatability, stability, and recovery of the analysis method are shown in Table 4. The findings showed that the proposed HPLC-DAD method could be used for the quantitative analysis of AAs.

\section{Sample analysis}

The proposed method was applied to quantify the aforementioned six alkaloids in raw and processed Aconiti kusnezoffii Radix from five sources (R1-R5 and P1-P5, respectively) (Table S1).

\subsection{Anti-inflammatory activity}

Effects of AEC and non-AEC on RAW264.7 cell viability

The effect of total alkaloids (AEC) and water (non-AEC) on the viability of RAW264.7 cells was evaluated using the 3-[4, 5-dimethyl-2-thiazolyl]-2, 5-diphenyl-2H-tetrazolium bromide (MTT) assay. RAW264.7 cells were incubated with increasing concentrations of AEC or non-AEC $(25-400 \mu \mathrm{g} / \mathrm{mL})$ for $24 \mathrm{~h}$. As shown in Figure 3A and 3B, AEC significantly decreased cell viability at concentrations of 100,200 , and $400 \mu \mathrm{g} / \mathrm{mL}$. Eventually, we selected $6.25,12.5,25$, and $50 \mu \mathrm{g} / \mathrm{mL}$ AEC and non-AEC for further investigations.

\section{Protective effects of AEC and non-AEC on LPS-treated RAW264.7 cells}

Compared with the LPS model group, the AEC-treated groups $(12.5,25$, and $50 \mu \mathrm{g} / \mathrm{mL}$ ) (Figure 3D) showed significantly improved inflammatory response, and a concentration-dependent effect was observed $(P<0.05)$. The results showed that AEC exerts good protective effects in the concentration range of $12.5-50 \mu \mathrm{g} / \mathrm{mL}$. Moreover, protective effects were observed in the non-AEC groups (same concentration) (Figure $3 \mathrm{C}$ ) only at a concentration of $12.5 \mu \mathrm{g} / \mathrm{mL}$. The NO assay clearly showed that LPS increased NO production, whereas AEC decreased NO production in LPS-treated RAW264.7 cells.

\section{Discussion}

Currently, studies on the chemical constituents of Aconiti kusnezoffii Radix are relatively simple (Zhi et al., 2020). These studies have only reported that Aconiti kusnezoffii Radix contains alkaloids and that the content of alkaloids changes before and after processing to reduce toxicity. There has been no further research

Table 3. Linear range, linearity equation, correlation coefficient, limit of detection (LOD), and limit of quantitation (LOQ) of the six analytes.

\begin{tabular}{cccccc}
\hline Analyte & $\begin{array}{c}\text { Linear } \\
\text { Range } \\
(\mu \mathrm{g} / \mathrm{mL})\end{array}$ & $\begin{array}{c}\text { Linearity } \\
\text { Equation }\end{array}$ & $\mathrm{R}^{2}$ & $\begin{array}{c}\text { LOD } \\
(\mu \mathrm{g} / \mathrm{mL})\end{array}$ & $\begin{array}{c}\text { LOQ } \\
(\mu \mathrm{g} / \mathrm{mL})\end{array}$ \\
\hline BAC & $1.463-292.5$ & $\mathrm{Y}=0.1591 \mathrm{X}-0.1166$ & 0.9999 & 0.731 & 2.925 \\
BMA & $1.090-218.0$ & $\mathrm{Y}=0.1499 \mathrm{X}+0.1773$ & 0.9995 & 0.545 & 2.180 \\
BHA & $1.120-224.0$ & $\mathrm{Y}=0.1526 \mathrm{X}-0.1586$ & 0.9992 & 1.120 & 5.600 \\
AC & $1.395-139.5$ & $\mathrm{Y}=0.1268 \mathrm{X}-0.1055$ & 0.9998 & 1.395 & 3.488 \\
MA & $1.560-156.0$ & $\mathrm{Y}=0.1355 \mathrm{X}-0.0171$ & 0.9998 & 1.560 & 3.900 \\
HA & $1.965-196.5$ & $\mathrm{Y}=0.1461 \mathrm{X}+0.0462$ & 0.9999 & 1.965 & 9.825 \\
\hline
\end{tabular}


on the alkaloids and their contents. Plants of the same genus have similar chemical constituents owing to their genetic relationships. Aconiti kusnezoffii Radix, Aconiti Radix, and Aconiti Lateralis Radix Praeparata are from the genus Aconitum. Therefore, the research data of Aconiti Radix and Aconiti Lateralis Radix Praeparata can be used as a reference to analyze the chemical
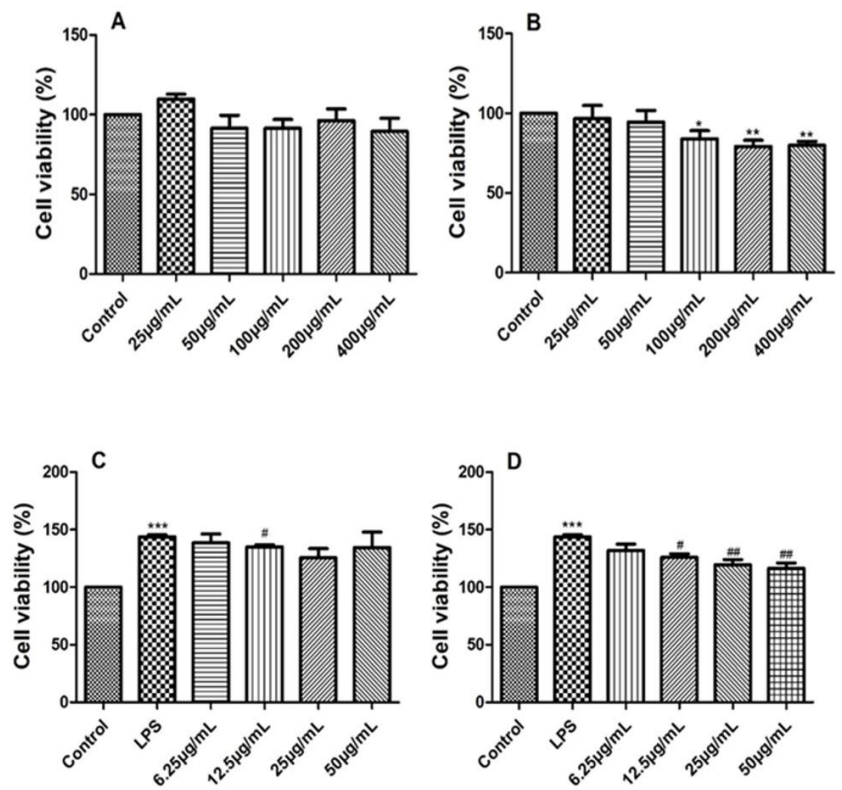

Figure 3. Effect of non-AEC and AEC on RAW264.7 cell viability and effect of non-AEC and AEC on RAW264.7 cell NO production. (A. non-AEC, B. AEC, compared with the normal group, ${ }^{\star} P<0.05$, ${ }^{* *} P<0.01$. C. non-AEC, D. AEC, compared with the normal group, *** $\mathrm{P}<0.001$, compared with the LPS group, \# $\mathrm{P}<0.05$, \#\# $\mathrm{P}<0.01$ ). composition of raw and processed Aconiti kusnezoffii Radix. On this basis, our study findings can be further expanded to identify the chemical constituents of Aconiti kusnezoffii Folium, Aconiti kusnezoffii Flos, and Aconiti kusnezoffii buds, which are commonly used in Mongolian medicine and have the same origin as Aconiti kusnezoffii Radix, in order to promote the clinical use of Aconiti kusnezoffii Radix in TCM and Mongolian medicine. The qualitative and quantitative analyses of the raw and processed Aconiti kusnezoffii Radix revealed that the kind, quantity, and content of alkaloids were different before and after processing (see Table S2). There are 64 alkaloids in both raw and processed Aconiti kusnezoffii Radix, 74 alkaloids in only raw Aconiti kusnezoffii Radix, and 17 alkaloids in only processed Aconiti kusnezoffii Radix. The analysis results of six alkaloids revealed that before processing, the content of DDAs (AC, MA, and $\mathrm{HA}$ ) was higher than that of MDAs (BAC, BMA, and BHA). After processing, the content of DDAs decreased whereas the content of MDAs increased. It can be inferred that some DDAs may be converted to MDAs after processing, which implies that processing Aconiti kusnezoffii Radix reduces its toxicity.

In the process of identification of chemical constituents of Aconiti kusnezoffii Radix by HPLC-MS/MS, we found that the retention time is related to the molecular weight and the molecular weight is closely related to the structure. The chromatographic peaks are divided into three parts, ADAs with $\mathrm{m} / \mathrm{z}$ ranging from 300 to 500 and retention time from 1 to $15 \mathrm{~min}$, MDAs and DDAs with $\mathrm{m} / \mathrm{z}$ ranging from 500 to 700 and retention time from 15 to $22 \mathrm{~min}$, and LDAs with $\mathrm{m} / \mathrm{z}$ ranging from 700 to 900 and retention time from 22 to $40 \mathrm{~min}$ (see Figure 2). The polarity of LDA is relatively low due to the presence of one or two fatty acid chains in the structure. Thus, the LDA is eluted last from the reversed-phase C18 column.

Table 4. Precision, recovery, repeatability, and stability of the six analytes.

\begin{tabular}{|c|c|c|c|c|c|c|c|}
\hline Analyte & $\begin{array}{c}\text { Concentration } \\
\text { level }\end{array}$ & $\begin{array}{l}\text { Intraday } \\
\text { Precision } \\
\text { (RSD, \%) }\end{array}$ & $\begin{array}{l}\text { Interday } \\
\text { Precision } \\
(\mathrm{RSD}, \%)\end{array}$ & $\begin{array}{c}\text { Recovery } \\
\text { Measurerd (\%) }\end{array}$ & RSD (\%) & $\begin{array}{c}\text { Repeatability } \\
(\mathrm{RSD}, \%, \mathrm{n}=6)\end{array}$ & $\begin{array}{c}\text { Stability } \\
\text { (RSD, \%, 24h) }\end{array}$ \\
\hline \multirow[t]{3}{*}{ BAC } & Low & 2.95 & 3.21 & 103.43 & 3.59 & & \\
\hline & Medium & 1.39 & 2.77 & 105.25 & 4.36 & 3.19 & 2.15 \\
\hline & High & 0.89 & 2.98 & 100.37 & 3.21 & & \\
\hline \multirow[t]{3}{*}{ BMA } & Low & 3.36 & 3.32 & 108.08 & 4.40 & & \\
\hline & Medium & 2.57 & 1.21 & 102.44 & 2.77 & 4.79 & 4.71 \\
\hline & High & 1.42 & 2.02 & 98.56 & 3.56 & & \\
\hline \multirow[t]{3}{*}{ BHA } & Low & 2.21 & 3.31 & 103.23 & 2.68 & & \\
\hline & Medium & 1.78 & 2.18 & 97.23 & 4.11 & 2.84 & 5.10 \\
\hline & High & 3.22 & 1.09 & 104.13 & 3.20 & & \\
\hline \multirow[t]{3}{*}{$\mathrm{AC}$} & Low & 2.68 & 0.45 & 95.37 & 2.91 & & \\
\hline & Medium & 2.23 & 1.31 & 106.29 & 2.63 & 1.66 & 2.78 \\
\hline & High & 1.79 & 2.77 & 100.53 & 1.78 & & \\
\hline \multirow[t]{3}{*}{ MA } & Low & 2.92 & 2.89 & 93.26 & 3.98 & & \\
\hline & Medium & 2.04 & 3.66 & 102.74 & 2.89 & 2.27 & 4.38 \\
\hline & High & 1.87 & 2.47 & 103.18 & 1.31 & & \\
\hline \multirow[t]{3}{*}{ HA } & Low & 3.33 & 2.13 & 104.78 & 3.33 & & \\
\hline & Medium & 1.12 & 1.68 & 98.99 & 2.87 & 1.31 & 1.43 \\
\hline & High & 2.39 & 3.55 & 107.27 & 1.36 & & \\
\hline
\end{tabular}


The structure of ADA, MDA, and DDA contain a large number of methoxyl and hydroxyl groups, and $\mathrm{MeOH}$ (32 Da), $\mathrm{H} 2 \mathrm{O}(18 \mathrm{Da})$, and $\mathrm{CO}(28 \mathrm{Da})$ are often absent in the secondary fragment ions. However, the LDAs are different from ADAs, MDAs, and DDAs, and are formed by the combination of alkaloid skeleton and fatty acid chain. Therefore, the LDA molecular composition can be hypothesized with the following rule.

$$
M F(L D A)=M F(A B S)+M F(F A)-\mathrm{H}_{2} \mathrm{O}
$$

Orbitrap MS, as a high-resolution MS, provides accurate information on the elemental composition of molecular ion peaks, which is the basis for the identification of isomers. The presence of an isomer is a common phenomenon in MS. Only a small part of the isomers can be distinguished by $\mathrm{tR}$, polarity, reference, precise molecular weight, and secondary fragment ions, but distinguishing a major part of the isomers using MS data remains a challenge. In our study, three same molecular weight peaks occurred at 29.78 (compound 127), 31.45 (compound 128), and $33.39 \mathrm{~min}$ (compound 141) in the TIC of $\mathrm{m} / \mathrm{z} 850$. For compound 127 , the protonated ion was at $\mathrm{m} / \mathrm{z} 850.5100(\mathrm{C} 49 \mathrm{H} 72 \mathrm{O} 11 \mathrm{~N},-0.94 \mathrm{ppm})$, which easily lost the hodt unit $(\mathrm{C} 18 \mathrm{H} 30 \mathrm{O} 3$, hydroxyoctadecatrienoic acid), producing the base product ion at $\mathrm{m} / \mathrm{z} 556[\mathrm{M}+\mathrm{H}$-hodt $]+$. The distinctive MS2 spectrum $(524[\mathrm{M}+\mathrm{H}$-hodt-MeOH]+, $496[\mathrm{M}+\mathrm{H}$-hodt$\mathrm{MeOH}-\mathrm{CO}]+$, and $464[\mathrm{M}+\mathrm{H}$-hodt- $2 \times \mathrm{MeOH}-\mathrm{CO}]+)$ was the same as the MS2 spectrum of BHA. Thus, compound 127 was identified as 8-hodt-BHA. For compound 128 , the $[\mathrm{M}+\mathrm{H}]+$ ion at $\mathrm{m} / \mathrm{z} 850.5100(\mathrm{C} 49 \mathrm{H} 72 \mathrm{O} 11 \mathrm{~N},-0.89 \mathrm{ppm})$ lost the linolen unit $(\mathrm{C} 18 \mathrm{H} 30 \mathrm{O} 2$, linolenic acid), producing the base product ion at $\mathrm{m} / \mathrm{z} 572[\mathrm{M}+\mathrm{H}$-linolen $]+$. The rest of the MS2 spectrum $(540[\mathrm{M}+\mathrm{H}$-linolen- $\mathrm{MeOH}]+, 512[\mathrm{M}+\mathrm{H}$-linolen-MeOH$\mathrm{CO}]+$, and $480[\mathrm{M}+\mathrm{H}-$ linolen- $2 \times \mathrm{MeOH}-\mathrm{CO}]+)$, was similar to the MS2 spectrum of BMA. Therefore, it was identified as 8-linolen-BMA. For compound 141 , the $[\mathrm{M}+\mathrm{H}]+$ ion at $\mathrm{m} / \mathrm{z}$ $850.5464(\mathrm{C} 50 \mathrm{H} 76 \mathrm{O} 10 \mathrm{~N},-2.55 \mathrm{ppm})$ lost the lino unit $(\mathrm{C} 18 \mathrm{H} 32 \mathrm{O} 2$, linoleic acid), producing the base product ion at $\mathrm{m} / \mathrm{z} 570[\mathrm{M}+\mathrm{H}-$ lino] + . The rest of the MS2 spectrum (538 [M+H-lino- $\mathrm{MeOH}]+$, $510[\mathrm{M}+\mathrm{H}-$ lino- $\mathrm{MeOH}-\mathrm{CO}]+$, and $478[\mathrm{M}+\mathrm{H}-\mathrm{lino}-2 \times \mathrm{MeOH}-$ $\mathrm{CO}]+$ ) was the same as the MS2 spectrum of DBA. Therefore, it was identified as 8-lino-DBA.

Aconiti kusnezoffii Radix has the functions of dispelling wind, removing dampness, and relieving pain. Similar to Aconiti Radix, it is used to treat rheumatism and arthritis. Aconiti Radix has significant anti-inflammatory activity. To clarify whether Aconiti kusnezoffii Radix has anti-inflammatory activity and identify chemical components that lead to the anti-inflammatory activity, further in-depth research is required. In the analysis of the chemical constituents of Aconiti kusnezoffii Radix, we found that it contains numerous alkaloids. Therefore, it is not clear whether the alkaloid part or the non-alkaloid part exerts the anti-inflammatory effects. Here, we isolated AECs and non-AECs and examined the effects on cell survival and $\mathrm{NO}$ release in a LPS-induced Raw264.7 inflammatory cell model. The results showed that AEC at concentrations between 12.5 and $50 \mu \mathrm{g} / \mathrm{mL}$ could significantly reduce the release of NO. In the future, we will verify the anti-inflammatory activity using zebrafish in vivo.

\section{Conclusions}

Here, using the established UHPLC-Q Exactive MS/MS and HPLC-DAD method, we identified 155 and quantified 6 components of raw and processed Aconiti kusnezoffii Radix. This method can be used to control the quality of Aconiti kusnezoffii Radix. Moreover, the method has the following advantages: high speed, simplicity, and low solvent consumption. Therefore, this novel method can be used for the qualitative and quantitative analyses of Aconitum products, such as Aconiti kusnezoffii Radix, Aconiti Radix, and Aconiti Lateralis Radix Praeparata. Furthermore, AEC exhibited good anti-inflammatory effects. Our findings provide a theoretical basis for the use of Aconiti kusnezoffii Radix to treat inflammatory diseases. However, further studies on the action mechanism and targets of Aconiti kusnezoffii Radix are required.

\section{References}

Ao, W. L. J., \& Buhe, B. T. (2013). Traditional mongolian medicine and prescription (p. 90). Chifeng: Inner Mongolia Science and Technology Press.

Borcsa, B., Csupor, D., Forgo, P., Widowitz, U., Bauer, R., \& Hohmann, J. (2011). Aconitum lipo-alkaloids--semisynthetic products of the traditional medicine. Natural Product Communications, 6(4), 527-536. http://dx.doi.org/10.1177/1934578X1100600413. PMid:21560765.

Li, S., Li, R., Zeng, Y., Meng, X. L., Wen, C. B., \& Zheng, S. C. (2019). Chemical components and pharmacological action of Aconiti Radix. Zhongguo Zhongyao Zazhi, 44(12), 2433-2443. PMid:31359708.

Liang, Y., Wu, J. L., Leung, E. L. H., Zhou, H., Liu, Z. Q., Yan, G. Y., Liu, Y., Liu, L., \& Li, N. (2016). Identification of Oxygenated Fatty Acid as a Side Chain of Lipo-Alkaloids in Aconitum carmichaelii by UHPLC-Q-TOF-MS and a Database. Molecules (Basel, Switzerland), 21(4), 437-454. http://dx.doi.org/10.3390/molecules21040437. PMid:27043515.

Liu, J., Pan, J. K., Wang, Y. P., Lin, D. K., Shen, D., Yang, H. J., Li, X., Luo, M. H., \& Cao, X. W. (2013). Component analysis of Chinese medicine and advances in fuming-washing therapy for knee osteoarthritis via unsupervised data mining methods. Journal of Traditional Chinese Medicine, 33(5), 686-691. http://dx.doi.org/10.1016/S02546272(14)60043-1. PMid:24660597.

National Pharmacopoeia Committee (2015). Pharmacopoeia of the People's Republic of China. Part 1 (pp. 236-237). Beijing: Chemical Industry Press.

Pang, B. Y., Zhu, Y., Lu, L. Q., Gu, F. B., \& Chen, H. L. (2016). The applications and features of liquid chromatography-mass spectrometry in the analysis of traditional chinese medicine. Evidence-Based Complementary and Alternative Medicine, 2016, 3837270. http:// dx.doi.org/10.1155/2016/3837270. PMid:27956918.

Song, Y. L., Zhang, N., Jiang, Y., Li, J., Zhao, Y. F., Shi, S. P., \& Tu, P. F. (2015). Simultaneous determination of aconite alkaloids and ginsenosides using online solid phase extraction hyphenated with polarity switching ultra-high performance liquid chromatography coupled with tandem mass spectrometry. RSC Advances, 5(9), 64196428. http://dx.doi.org/10.1039/C4RA14088E.Wang, C. Y., Jang, H. J., Han, Y. K., Su, X. D., Lee, S. W., Rho, M. C., Wang, H. S., Yang, S. Y., \& Kim, Y. H. (2018a). Alkaloids from Tetrastigma hemsleyanum and Their Anti-Inflammatory Effects on LPS-Induced RAW264.7 Cells. Molecules (Basel, Switzerland), 23(6), 1445-1455. http://dx.doi. org/10.3390/molecules23061445. 
Wang, D., Jia, D. X., Li, Z. Z., Yao, J. K., Zhang, L., Sun, X. B., Gao, X. M., \& Wang, J. X. (2018b). Safety evaluation and risk control measures for Aconiti Kusnezoffii Radix. Zhongguo Zhongyao Zazhi, 43(15), 3093-3100. PMid:30200703.

Wang, F., Yue, Z. G., Xie, P., Zhang, L., Li, Z., Song, B., Tang, Z. S., \& Song, X. M. (2016). C19-Norditerpenoid Alkaloids from Aconitum szechenyianum and Their Effects on LPS-Activated NO Production. Molecules (Basel, Switzerland), 21(9), 1175-1182. http://dx.doi. org/10.3390/molecules21091175.

Wang, F. P., Chen, Q. H., \& Liang, X. T. (2009). The C18-diterpenoid alkaloids. The Alkaloids. Chemistry and Biology, 67, 1-78. http:// dx.doi.org/10.1016/S1099-4831(09)06701-7. PMid:19827365.

Wang, F. P., Chen, Q. H., \& Liu, X. Y. (2010). Diterpenoid alkaloids. Natural Product Reports, 27(4), 529-570. http://dx.doi.org/10.1039/ b916679c. PMid:20336236.

Wang, F. P., \& Liang, X. T. (2002). C20-diterpenoid alkaloids. The Alkaloids. Chemistry and Biology, 59, 1-280. http://dx.doi.org/10.1016/ S0099-9598(02)59008-8. PMid:12561418.

Xu, W., Zhang, J., Zhu, D. Y., Huang, J., Huang, Z. H., Bai, J. Q., \& Qiu, X. H. (2014). Rapid separation and characterization of diterpenoid alkaloids in processed roots of Aconitum carmichaeli using ultra high performance liquid chromatography coupled with hybrid linear ion trap-Orbitrap tandem mass spectrometry. Journal of Separation Science, 37(20), 2864-2873. http://dx.doi.org/10.1002/ jssc.201400365. PMid:25124198.
Yue, H., Pi, Z. F., Song, F. R., Liu, Z. Q., Cai, Z. W., \& Liu, S. Y. (2009). Studies on the aconitine-type alkaloids in the roots of Aconitum Carmichaeli Debx. by HPLC/ESIMS/MS(n). Talanta, 77(5), 18001807. http://dx.doi.org/10.1016/j.talanta.2008.10.022. PMid:19159802.

Zhang, M., Peng, C. S., \& Li, X. B. (2015). In vivo and in vitro metabolites from the main diester and monoester diterpenoid alkaloids in a traditional chinese herb, the aconitum species. Evidence-Based Complementary and Alternative Medicine, 2015, 252434. PMid:25705235.

Zhang, N., Song, Y. L., Song, Q. Q., Shi, S. P., Zhang, Q., Zhao, Y. F., Li, J., \& Tu, P. F. (2016). Qualitative and quantitative assessments of Aconiti Lateralis Radix Praeparata using high-performance liquid chromatography coupled with diode array detection and hybrid ion trap-time-of-flight mass spectrometry. Journal of Chromatographic Science, 54(6), 888-901. http://dx.doi.org/10.1093/chromsci/bmv245. PMid:27048641.

Zhang, Z., Jiang, M. Y., Wei, X. Y., Shi, J. F., Geng, Z., Yang, S. S., Fu, C. M., \& Guo, L. (2019). Rapid discovery of chemical constituents and absorbed components in rat serum after oral administration of Fuzi-Lizhong pill based on high-throughput HPLC-Q-TOF/ MS analysis. Chin Med-UK, 14(1), 6-27. http://dx.doi.org/10.1186/ s13020-019-0227-z.

Zhi, M. R., Gu, X. R., Han, S., Liu, K. Y., Liu, Z. Q., Tang, Y. N., Han, X. T., Li, F., Yang, Z. G., Tan, P., Zhao, H. Y., \& Du, H. (2020). Chemical variation in Aconti Kusnezoffii Radix before and after processing based on UPLC-Orbitrap-MS. Zhongguo Zhongyao Zazhi, 45(5), 1082-1089. PMid:32237450. 


\section{Supplementary Materials}

The online version of this article contains Supplementary Materials.

Table S1: Content $(\mu \mathrm{g} / \mathrm{g})$ of the six alkaloids in five batches of raw and processed Aconiti kusnezoffii Radix.

Table S2: Precursor and product ions of the constituents in the $70 \% \mathrm{MeOH}$ extract of raw and processed Aconiti kusnezoffii Radix in the UHPLC-ESI+-MS/MS experiments.

This material is available as part of the online article from http://www.scielo.br/cta 